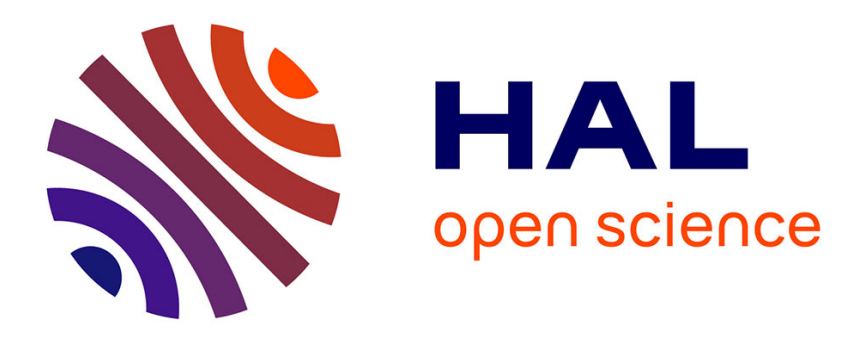

\title{
Personal verification system based on retina and SURF descriptors
}

Takwa Chihaoui, Hejer Jlassi, Rostom Kachouri, Kamel Hamrouni, Mohamed

Akil

\section{- To cite this version:}

Takwa Chihaoui, Hejer Jlassi, Rostom Kachouri, Kamel Hamrouni, Mohamed Akil. Personal verification system based on retina and SURF descriptors. 13th IEEE International Multi-Conference on Systems, Signals \& Devices (SSD 2016), Mar 2016, Leipzig, Germany. 10.1109/SSD.2016.7473709 . hal-01305985

\section{HAL Id: hal-01305985 \\ https://hal.science/hal-01305985}

Submitted on 22 Apr 2016

HAL is a multi-disciplinary open access archive for the deposit and dissemination of scientific research documents, whether they are published or not. The documents may come from teaching and research institutions in France or abroad, or from public or private research centers.
L'archive ouverte pluridisciplinaire HAL, est destinée au dépôt et à la diffusion de documents scientifiques de niveau recherche, publiés ou non, émanant des établissements d'enseignement et de recherche français ou étrangers, des laboratoires publics ou privés. 


\title{
PERSONAL VERIFICATION SYSTEM BASED ON RETINA AND SURF DESCRIPTORS
}

\author{
Takwa Chihaoui* ${ }^{\dagger}$, Hejer Jlassi $^{\dagger}$, Rostom Kachouri* ${ }^{*}$ Kamel Hamrouni ${ }^{\dagger}$ and Mohamed Akil* \\ *Université Paris-Est, Laboratoire Informatique Gaspard-Monge, \\ Equipe A3SI, ESIEE Paris, France \\ Email: takwa.chihaoui@esiee.fr, rostom.kachouri@esiee.fr, mohamed.akil@esiee.fr \\ †Université de Tunis El Manar, Ecole Nationale d'Ingénieurs de Tunis \\ LR-SITI Signal Image et Technologie de l'information, Tunis, Tunisie \\ Email: hejer_enit@enit.rnu.tn, kamel.hamrouni@enit.rnu.tn
}

\begin{abstract}
Today, Human recognition, especially based on retina, has been an important and attractive topic of scientific research. Most efforts in Biometrics tend to develop more efficient systems which compromise speed and robustness of authentication. In fact, retinal images often suffer from imperfections such as background intensity variation, affine transformations (translation, rotation, scale changes) variations from pattern to other. These defects can seriously affect features extraction in terms of quality and execution time. In this context, in order to overcome these defects, we propose in this paper a novel retinal verification system based on the Speeded Up Robust Features (SURF) extraction. This feature extraction method is so fast and invariant to the affine transformations such as rotation, scale changes and translation. We employ the Optical Disc interest Ring (ODR) method as a preprocessing step in order to further speed up the system and improve the performance. A subset of the VARIA database is used to evaluate the proposed SURF based system. It compromises a high quality with $100 \%$ of verification accuracy rate and a time processing very lower than existing verification systems.

Index Terms - Biometrics, Retinal verification systems, Optical Disc interest Ring (ODR) method, Speeded up Robust Features (SURF)
\end{abstract}

\section{INTRODUCTION}

Automatic Human recognition has been an important and attractive topic of scientific research. Indeed, biometric authentication gets the leading role, since it cannot be borrowed, stolen or forgotten and forging is practically impossible. It is used as the most secure recognition tool. Biometrics is the automatic recognition of a person based on his distinguishing features. In Fact, these are all physical or personal traits automatically measurable, robust and distinctive features that can be used to identify an individual or verify the claimed identity of an individual [1]. Various biometric systems have been developed based on fingerprint, palmprint, hand geometry, vein, face, iris, retina and ear. Retinal recognition has received increasing attention in recent years as it provides promising solution to security issues [2] due to its uniqueness, universality, time-invariance and difficulty to be forged. Retinal recognition is a relatively new approach, compared to other biometric features. It includes two modes: Identification which compare each pattern to all others $(1: N)$ and Verification which compare the pattern to others from the same individual $(1: 1)$. In this paper, we are interested in the verification mode. Since retinal patterns have highly distinctive traits, the features extracted from retina identify effectively persons, even among genetically identical twins. This pattern will not change through the life of the individual, unless a serious pathology appears in the eye. Most common diseases such as diabetes do not change the pattern in a way that its topology is affected. Hence, this is one of the most secure and valid biometric trait for personal authentication. However, Retinal verification systems can suffer from some problems. Due to the acquisition process of the retinography, retinal images often suffer from imperfections such as background intensity variation, affine transformations (translation, rotation, scale changes) variations from pattern to other. These defects can seriously affect features extraction. In order to overcome these imperfections, we propose, in this paper, a new approach for verifying retina images based on the ODR preprocessing and Speeded Up Robust Features (SURF) description. The verification task in the proposed system is invariant from affine transformations such as rotation, scale changes and translation, and illumination variations. The rest of this paper is organized as follows: Section 2 introduces some existing retinal verification systems. Section 3 presents the proposed system. The pre-processing stage, feature extraction procedure and decision making process is discussed in this Section. Experimental results are presented in Section 4. conclusion is given in the last section.

\section{RETINAL VERIFICATION SYSTEMS: RELATED WORKS}

Many retina authentication systems have been exploited in order to find the most secure biometric one. $\mathrm{Xu}$ et al. [3] proposed a new method for recognition. First, they extracted blood vessel skeleton feature using contrast-limited adaptive histogram equalization. After the filtering treatment and extracting shape feature, shape curve of blood vessels was obtained. Shape curve matching was later carried out by means of reference point matching. In their method for recognition, feature matching consisted of finding affine transformation parameters which relates the query image and its best corresponding enrolled image. The computational time of this method is high because a number of parameters should be computed for all possible correspondences between the input 
and reference images in the dataset. Ortega et al. [4] proposed a novel personal verification system based on creases-based method. The feature extraction step consists of generating a Creases image representing the main vessels in order to extract landmarks. After that, they employed a registration process based on the similarity transformation (ST), in order match the reference pattern from the database and the input one. When the biometric patterns were aligned, they measured the degree of similarity by means of a similarity metric to correctly classify authentications in both classes: attacks and clients. Marino et al. [5] introduced a novel verification system based on retinal vessel tree pattern. In that work, the whole vascular tree structure was used as the feature pattern. The results showed a high confidence band in the authentication process but with a small database which included only 6 individuals with 2 images for each of them. One of the weak points of this proposed system was the very high computation time of storing and handling a whole image as the biometric pattern. Oinonen et al. [6] proposed a novel method for verification based on minutiae features. The proposed method consisted of three steps: blood vessel segmentation, feature extraction, and feature matching. In practice, vessel segmentation can be viewed as a preprocessing phase for feature extraction. Then, vessel crossings and their orientation information were obtained. These data were matched with the corresponding ones from the comparison image. The method used the vessel direction information for improved matching robustness. The computational time of this method for segmentation, feature extraction, and matching is high. Amin Dehghani et al. [7] proposed a new human recognition (identification and verification) method based on Harris corners and similarity function of retinal images without using vessel segmentation methods. As a preliminary step, they compensated the rotation angle of head or eye movement in front of the retina fundus camera using phase correlation technique. After that, they detected corners in the retina image using Harris corner detector and pruning algorithm. The weakness of this system is that it may be not effective when retinal image are affected by some pathologies. Arathi Arakala et al. [8] proposed an automatic retina verification framework. First, they extracted the vascular pattern of retinal image using a family of matched filters in the frequency domain and morphological operators. Then, they created a spatial graph template based on the extracted pattern. Finally, they compared between two graphs derived from two retinal images using a biometric graph matching (BGM) algorithm which is robust to translation, nonlinear distortion, and small rotations. As a decision step, this system returns a set of distance measures between them in order to distinguish between genuine and imposter comparisons. A common problem on previous approaches is that are variant to affine transformations such as translation, rotation and scale changes, which necessitate a registration phase. This additional phase consists of align the target images with the reference image. Moreover, most of existing approaches suffer from an important execution time. So, in this work, the use of Speeded Up Robust Features (SURF) for feature extraction aims prin- cipally to eliminate the registration phase and consequently to reduce the processing time of the verification system.

\section{PROPOSED SURF-BASED RETINAL VERIFICATION SYSTEM}

Depending on several factors, such as the eye location in the objective, patterns may suffer from some deformations. A reliable and efficient model is necessary to deal with these deformations allowing transforming the candidate pattern in order to get a pattern similar to the reference one. The movement of the eye in the image acquisition process basically consists in translation in axis, rotation and sometimes a very small change in scale. In this work, we propose a novel retinal verification system based on the Speeded Up Robust Features (SURF) feature extraction. In the proposed system, segmentation methods that increase computational time were not used. In order to improve the quality and reduce the processing time, a pre-processing step based on the ODR method that enhances input retinal images and extracts an interest ring around the optical disc is needed (Section A). Next, the fast extraction of SURF feature points is explained in Section B. The final step in the verification process is the matching between the keypoints from the reference image and the keypoints from the acquired image based on the test g2NN (Section C).

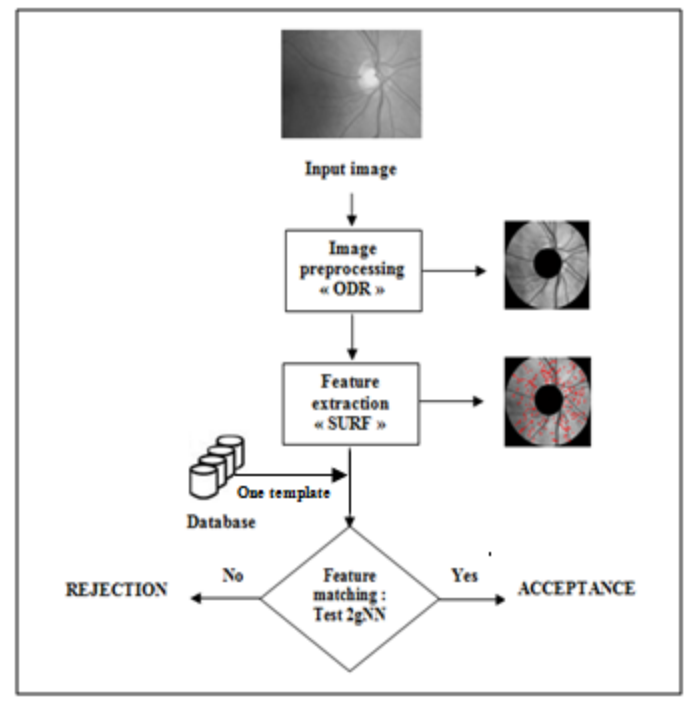

Fig. 1. Flowchart of our proposed retinal system.

\section{A. Ring extraction around the optical disc}

On the one hand, the presence of noise, the low contrast between vasculature and background, brightness, and the variability of vessel diameter, shape and orientation are the main obstacles in retinal images. On the other hand, SURF characterization is based on the intensity variation between regions, which requires the preservation of the brightest area (the optical disc). In order to overcome these problems, we use our recently proposed method Optical Disc interest Ring (ODR) [9] for preprocessing the retinal image in order to 
improve its quality and extract an interest ring centered at the center of the optical disc as an interest region of retinal image.

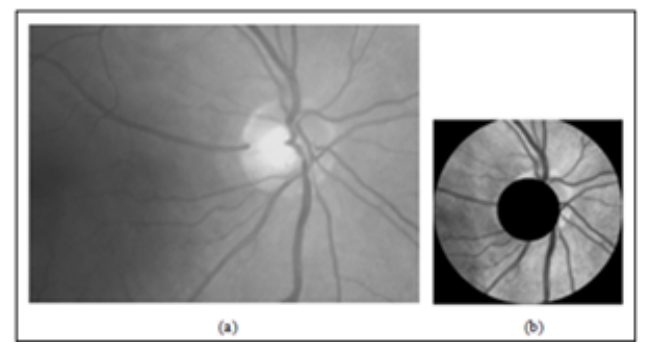

Fig. 2. The result of ODR preprocessing step: (a) Input retinal image and (b) the ring extracted around the optical disc.

\section{B. Feature extraction}

In this step, we apply the Speeded Up Robust Features transform (SURF) [10] to provide a feature description of the retinal image. The output of this step is a 64 elements feature vector for each detected point. SURF is an algorithm for detection of interest areas. It is similar to SIFT descriptor in terms of repeatability and robustness, but exceeds in terms of speed extraction and features vector comparison. Thus, this method is very fast in processing time and is invariant to scaling, rotation and translation. The SURF algorithm involves these steps: an integral representation of the image, a filtering of the image using the Hessian matrix, a suppression of nonmaxima points and a calculation of the descriptors using the Haar wavelet.

1) Integral representation of the image: SURF uses an alternative re-presentation of image called "Integral Image" to speed up the calculation of features. The result is an integral image that has identical dimensions to the input image.

2) Filtering using the Hessian matrix: This step aims on the detection of interest points using the determinant of the Hessian matrix. The Hessian matrix of 2 dimension shown on the equation (1) is a square matrix of Gaussian second order derivative with $x$ and $y$ direction of the image $f$, which is variations of the image around a point.

$$
H(f(x, y))=\left(\begin{array}{ll}
\frac{d f^{2}}{d x^{2}} & \frac{d f^{2}}{d x y} \\
\frac{d f^{2}}{d y x} & \frac{d f^{2}}{d y^{2}}
\end{array}\right)
$$

The approximated determinant of this Hessian matrix shown below use three filters to calculate the elements of the matrix $H(f(x, y))$. The use of the Hessian determinant becomes effective with an analysis scales, keeping only the interesting points independently of the observation scale.

3) Suppression of non-maxima: After calculating the Hessian responses SURF search the maxima points which are the interest points of the image on different scales. Then, the nonmaxima points are deleted.
4) Calculation of descriptors : The last step is to calculate the descriptor vector for each zone of interest. Indeed, for each sub-region $4 * 4$, we construct a vector $\mathrm{v}$ of 4 elements, defined as in equation (2):

$$
v=\left(\begin{array}{c}
\sum d x \\
\sum|d x| \\
\sum d y \\
\sum|d y|
\end{array}\right)
$$

This vector is a descriptor of the sub-region distribution of gradient. The 16 sub-regions vectors are then aggregated into a single vector of $16 * 4=64$ which is the descriptor of the region of interest. So, the size of the descriptor vector is 64 ,smaller than SIFT descriptor [9] in order to improve the speed comparison. The following figure 3 shows the SURF keypoints distribution on the preprocessed retinal image.

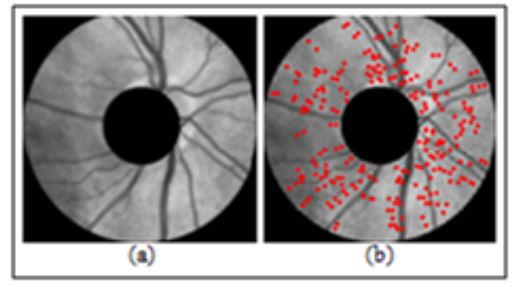

Fig. 3. SURF Keypoints distribution: (a) the input interest ring extracted and (b) the ring SURF keypoints characterized.

\section{Feature matching strategy}

This step of matching is performed in the SURF space among the feature vectors of each keypoint to identify similar regions in the sampled image. According to Lowe [11], he limit the classic approach to match keypoints which fixe a threshold on the Euclidean distance between descriptors because of the high-dimensionality of the feature space. However, he used a matching technique called g2NN test to find the best candidate which is based not only on the distance with the first most similar keypoint, but also with the second one; in particular, he used the ratio between the distance to the candidate match and the distance to the second similar feature point. The Principe of this test of matching is that the ratio between the distance of the candidate match di and the distance of the 2 nd nearest neighbor di+1 is low in the case of a match (e.g. lower than 0.6 ) and very high in case of two indiscriminate features (e.g. greater than 0.6). If $\mathrm{n}$ is the value in which the procedure stops, each keypoint in correspondence to a distance in $\mathrm{d} 1, \ldots, \mathrm{dk}$ where $(1<\mathrm{n}<\mathrm{m})$ is considered as a match for the examined key-point. Finally, by iterating over all keypoints, we can obtain the set of matched points. All the matched keypoints are retained to be linked in the next step. The following figure 4 illustrates an example of SURF keypoints matching between two images.

\section{EXPERIMENTAL RESULTS}

All the experiments are implemented in MATLAB, and performed on a PC with a $3.2 \mathrm{GHz}$ CPU and $4 \mathrm{G}$ Byte memory. 


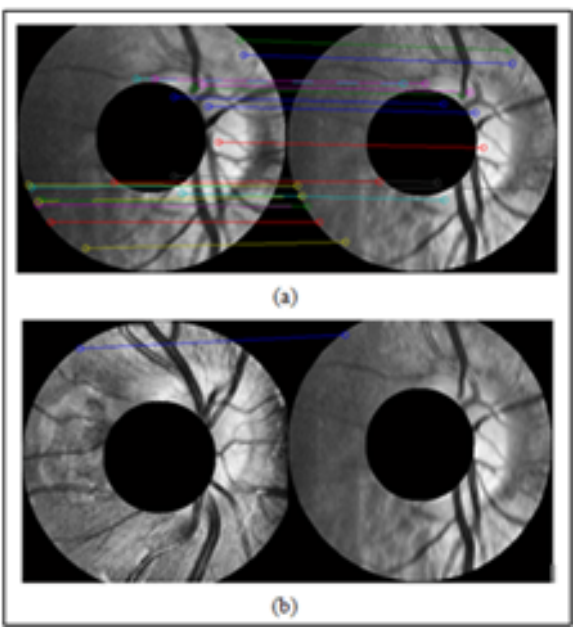

Fig. 4. Example of g2NN test based SURF keypoints between the clients (authorised) (a) and attacks (unauthorised) (b) authentication cases

To evaluate our retinal verification system, the VARIA [12] which is the largest currently available publicly database, is used for the experiment. It has 233 images from 139 people, with between one to seven samples per person. The images with only one sample and some samples that were identical to each other are excluded from the database. This left 135 retina images from 57 individuals including 42 with 2 samples, 10 with 3 samples, 4 with 4 samples and 1 with 5 samples. The lack of large publicly available retinal databases inhibits large scale and consistent testing of any retinal verification system. The Receiver Operating Characteristic (ROC) curve shows the measured accuracy performance of our biometric system. It illustrates the relationship between (1-FRR) and FAR is shown in Figure5. Our system provides also an AUC (Area Under Curve) value of $100 \%$.

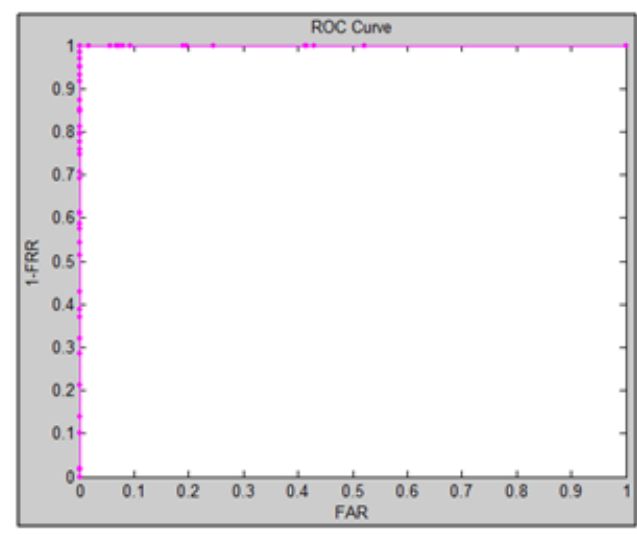

Fig. 5. The Receiver Operating Characteristic (ROC) curve of our proposed system.

Figure 6 shows the False Acceptance Rate (FAR) and the False Reject Rate (FRR) curves. A usual error measure in biometric verification system is the equal error rate (EER) that indicates the error rate where FAR curve and FRR curve are equal. EER is found from these curves as shown in Figure6. In order

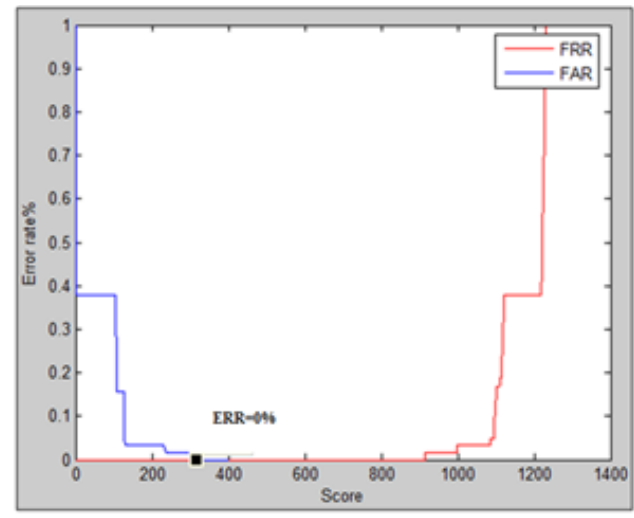

Fig. 6. The FRR and FAR curve of our SURF-based system.

to evaluate the efficiency of our proposed system in terms of quality, we compare it with some retinal verification systems [3] [4] as shown in Table I.

TABLE I

THE AUC RATE AVERAGE OF OUR PROPOSED RETINAL VERIFICATION SYSTEM AND EXISTING SYSTEMS.

\begin{tabular}{|l|cl|}
\hline Verification system & AUC value & $\begin{array}{l}\text { Equal Error } \\
\text { Rate (EER) }\end{array}$ \\
\hline Our SURF-based system & $100 \%$ & $0 \%$ \\
Ortego et al. [4] system & $100 \%$ & $0.01 \%$ \\
Xu et al. [3] system & $99.95 \%$ & $0.19 \%$ \\
Hannu et al. [6] system & $100 \%$ & $0 \%$ \\
Xianjing Meng et al. [7] system & $100 \%$ & $0 \%$ \\
\hline
\end{tabular}

As shown in Table1, our method verifies $100 \%$ of retinal images accurately, as Hannu et al. [6] and Xianjing Meng et al. [7] systems. This is due to the invariance to the rotation and translation ensured by SURF. As shown in table I, the Equal Error Rate (EER) of our system achieved the $0 \%$ however other systems [3] [4] suffer respectively from an error rate (Equal Error Rate) $0.19 \%$ and $0.01 \%$. The most gain of our proposed system compared to other verification systems is in term of processing time. In fact, as shown in table II, our system is the fastest one with an average of processing time of $0.03 \mathrm{~s}$, compared to respectively $\mathrm{Xu}$ et al. [3] system which is very consuming time with approximately $45 \mathrm{~s}$ and Xianjing Meng et al. [7] with 7.87s. The huge execution time of the system [3] is due to the complicate matching process which compute a number of rigid motion parameters should be computed for all possible correspondences between every the input and enrolled images.

TABLE II

THE AVERAGE PROCESSING TIME OF OUR VERIFICATION SYSTEM AND EXISTING SYSTEMS.

\begin{tabular}{|l|c|}
\hline Verification system & Average of Processing time \\
\hline Our SURF-based system & $0.03 \mathrm{~s}$ \\
Hannu et al. [6] system & $2.31 \mathrm{~s}$ \\
Xianjing Meng et al. [7] system & $7.87 \mathrm{~s}$ \\
Xu et al. [3] system & $45 \mathrm{~s}$ \\
\hline
\end{tabular}

Indeed, our reached processing time is essentially due to 
the fast SURF extraction step and the interest ring extraction, which assure minimum of SURF keypoints and consequently lead to faster matching process.

\section{CONClusion}

In this paper, we present an automatic retina verification system based on Surfing process. It is described as follows. Firstly, the pre-processing phase consists of the extraction of a disc interest region around the optical disc to be an input for the second phase. Secondly, the features of the extracted region are extracted by the SURF descriptor. Finally, the test g2NN is applied to compute the number of matched keypoint pairs and classify identical retinal images. Our experimental results show that this work compromises an exact personal verification and a very lower computational complexity. The accuracy of the proposed system for human verification is $100 \%$.

\section{REFERENCES}

[1] P.Padma Polash and M. Maruf Monwar, Human iris recognition for biometric identification, IEEE 10th international conference on Computer and information technology, pp. 1-5, 2007.

[2] R.B.Hill, Retinal identification, in Biometrics: Personal Identification in Networked Society, A.Jain, R.Bolle, and S.Pankati, Eds., p.126, Springer, Berlin, Germany, 1999.

[3] Z-W Xu, X-X Guo, X-Y Hu, X Cheng,The blood vessel recognition of ocular fundus, Proceedings of the 4th International Conference on Machine Learning and Cybernetics, pp. 44934498, 2005.

[4] Marcos Ortega, M.G.Penedo, J.Rouco, N.Barreira, M.J.Carreira Personal verification based on extraction and characterization of retinal feature points, Journal of Visual Languages and Computing, pp.80-90, 2009.

[5] C. Marino, M. G. Penedo, M. Penas, M. J. Carreira, and F. Gonzalez, Personal authentication using digital retinal images, Pattern Analysis and Applications, vol. 9, no. 1, pp. 2133, 2006.

[6] Hannu Oinonen, Heikki Forsvik, Pekka Ruusuvuori, Olli YliHarja, Ville Voipio, and Heikki Huttunen, Identity verification based on vessel matching from fundus images, ICIP, pp.4089-4092. IEEE, 2010.

[7] Xianjing Meng, Yilong Yin, Gongping Yang and Xiaoming Xi. Retinal Identification Based on an Improved Circular Gabor Filter and Scale Invariant Feature Transform, In Sensors (14248220), Vol. 13 Issue 7,p9248, Jul 2013.

[8] A Dehghani, Z Ghassabi, HA Moghddam, and MS Moin, Human recognition based on retinal images and using new similarity function, EURASIP Journal on Image and Video Processing. 2013.

[9] Seyed Mehdi Lajevardi, Arathi Arakala, Stephen A Davis, and Kathy J Horadam, Retina Verification System Based on Biometric Graph Matching, IEEE Transactions on Image Processing; 22(9):3625-3635, 09/2013.

[10] Chihaoui Takwa, Kachouri Rostom, Jlassi Hejer, Hamrouni Kamel and Akil Mohamed, Human Identification System based on the detection of Optical Disc Ring in retinal images, IPTA2015, Orleans, France, 19-21 Nov 2015.

[11] Herbert Bay, Speeded-Up Robust Features (SURF), Computer Vision and Image Understanding 110, pp.346359, 2008.

[12] David G. Lowe, Distinctive Image Features from Scale-Invariant Keypoints, Computer Science Department, University of British Columbia, Vancouver, B.C., Canada, January 5, 2004.

[13] VARIA. VARPA. Retinal Images for Authentication (Database), http://www.varpa.es/varia.html 